\title{
Upper and lower solution method for Hilfer fractional evolution equations with nonlocal conditions
}

\author{
Haide Gou ${ }^{1 *}$ and Yongxiang $\mathrm{Li}^{1}$
}

${ }^{*}$ Correspondence: 842204214@qq.com 'Department of Mathematics, Northwest Normal University, Lanzhou, P.R. China

\section{Springer}

\begin{abstract}
This paper is concerned with the existence of extremal mild solutions for Hilfer fractional evolution equations with nonlocal conditions in an ordered Banach space $E$. By employing the method of lower and upper solutions, the measure of noncompactness, and Sadovskii's fixed point theorem, we obtain the existence of extremal mild solutions for Hilfer fractional evolution equations with noncompact semigroups. Finally, an example is provided to illustrate the feasibility of our main results.
\end{abstract}

MSC: 26A33; 34K30; 34K45; 47D06

Keywords: Lower and upper solution; Mild solutions; Hilfer fractional derivative; Noncompact measure

\section{Introduction}

In recent years, many authors began to consider Hilfer fractional differential equations, see [1-7]. Presently, Hilfer fractional evolution equations have also been widely dealt with by many scholars. In [2], Gu and Trujillo investigated a class of Hilfer fractional evolution equations and established the existence results of mild solutions to such issues, and then Furati et al. [8] considered an initial value problem for a class of Hilfer fractional differential equations.

Later, the nonlocal problems have had better effects in applications than the initial problem, many contributions have been made in applications of fractional evolution equations with nonlocal conditions, see $[7,9,10]$ and the references therein. For example, Liang and Yang [11] investigated the exact controllability of the nonlocal Cauchy problem for the fractional integro differential evolution equations in Banach spaces $E$ :

$$
\left\{\begin{array}{l}
D^{q} x(t)+A x(t)=f(t, x(t), G x(t))+B u(t), \quad t \in J, \\
x(0)=\sum_{k=1}^{m} c_{k} x\left(t_{k}\right)
\end{array}\right.
$$

where $D^{q}$ denotes the Caputo fractional derivative of order $q \in(0,1),-A: D(A) \subset E \rightarrow E$ is the infinitesimal generator of a $C_{0}$-semigroup $T(t)(t \geq 0)$ of uniformly bounded linear operators, $B$ is a linear bounded operator; $f$ is a given function and the operator is given

(c) The Author(s) 2019. This article is distributed under the terms of the Creative Commons Attribution 4.0 International License (http://creativecommons.org/licenses/by/4.0/), which permits unrestricted use, distribution, and reproduction in any medium, provided you give appropriate credit to the original author(s) and the source, provide a link to the Creative Commons license, and indicate if changes were made. 
by

$$
G x(t)=\int_{0}^{t} K(t, s) x(s) d s
$$

Over the past year, some recent papers investigated the existence of mild solutions for Hilfer fractional evolution equations with nonlocal conditions. In [3], Min Yang et al. studied the existence and uniqueness of mild solutions to the following Hilfer fractional evolution equations:

$$
\left\{\begin{array}{l}
D_{0+}^{v, \mu}[u(t)-h(t, u(t))]=A u(t)+f(t, u(t)), \quad t \in J^{\prime}=(0, b], \\
I_{0+}^{(1-v)(1-\mu)}[u(0)-h(0, u(0))]-g(u)=u_{0},
\end{array}\right.
$$

with the associated $C_{0^{-}}$-semigroup being compact or not, where $D_{0_{+}}^{v, \mu}$ denotes the Hilfer fractional derivative of order $\mu$ and type $v, 0 \leq v \leq 1,0<\mu<1$. In [5], Ahmed et al. studied the existence of mild solutions for Hilfer fractional stochastic integro-differential equations of the form

$$
\left\{\begin{array}{l}
D_{0+}^{v, \mu}[u(t)+F(t, v(t))]+A u(t)=\int_{0}^{t} G(s, \eta(s)) d \omega(s), \quad t \in J:=(0, b], \\
I_{0+}^{(1-v)(1-\mu)} u(0)-g(u)=u_{0},
\end{array}\right.
$$

where $\left.(t, v(t))=\left(t, u(t), u\left(b_{1}(t)\right)\right), \ldots, u\left(b_{m}(t)\right)\right)$ and $\left.(t, \eta(t))=\left(t, u(t), u\left(a_{1}(t)\right)\right), \ldots, u\left(a_{n}(t)\right)\right)$, $D_{0+}^{v, \mu}$ denotes the Hilfer fractional derivative $0 \leq v \leq 1,0<\mu<1,-A$ is the infinitesimal generator of an analytic semigroup of bounded linear operators $S(t), t \geq 0$ on a separable Hilbert space.

In [6], Ahmed et al. studied the existence and controllability results for nonlinear delay Hilfer fractional differential equation with impulsive condition of the form

$$
\left\{\begin{array}{l}
D_{0+}^{v, \mu} u(t)=A u(t)+f\left(t, u\left(\gamma_{1}(t)\right), \int_{0}^{t} h(t, s) g\left(s, u\left(\gamma_{2}(s)\right)\right) d s\right), \quad t \in J=(0, b], t \neq t_{k}, \\
\Delta u\left(t_{k}\right)=I_{k}\left(u\left(t_{k}^{-}\right)\right), \quad k=1,2, \ldots, m, \\
I_{0+}^{(1-v)(1-\mu)} u(0)=u_{0}
\end{array}\right.
$$

where $D_{0+}^{v, \mu}$ is the Hilfer fractional derivative, $A$ is the infinitesimal generator of a $C_{0^{-}}$ semigroup $T(t)$ on $E$.

On the other hand, by employing the method of lower and upper solutions to study the existence of an extremal mild solution for a class of fractional evolution equation is an interesting issue, which has been the focus of attention in [9, 10, 12-14]. In [14], Chen and $\mathrm{Li}$ used the monotone iterative method and lower and upper solutions method to discuss the existence and uniqueness of mild solutions for a class of semilinear evolution equations with nonlocal conditions in an ordered Banach space $E$ :

$$
\left\{\begin{array}{l}
u^{\prime}(t)+A u(t)=f(t, u(t)), \quad t \in J=[0, b] \\
u(0)=\sum_{k=1}^{p} c_{k} u\left(t_{k}\right)+u_{0}
\end{array}\right.
$$

where $A: D(A) \subset E \rightarrow E$ is a closed linear operator and $-A$ generates a $C_{0}$-semigroup $T(t)(t \geq 0)$ on $E, f \in C(J \times E, E), J=[0, b], b>0$ is a constant, $0<t_{1}<t_{2}<\cdots<t_{b}, p \in \mathbb{N}, c_{k}$ are real numbers, $c_{k} \neq 0, k=1,2, \ldots, p, u_{0} \in E$. 
In [15], Vikram Singh et al. investigated the existence and uniqueness of mild solutions for Sobolev type fractional impulsive differential systems with nonlocal conditions

$$
\left\{\begin{array}{l}
{ }^{c} D^{\beta}[B u(t)]=A u(t)+f\left(t, u(t), \int_{0}^{t} K(t, s, u(s)) d s\right), \quad t \in J=[0, a], t \neq t_{j}, \\
\left.\Delta u\right|_{t=t_{j}}=I_{j}\left(u\left(t_{j}\right)\right), \quad j=1,2, \ldots, m, m \in \mathbb{N}, \\
{ }^{L} D^{1-\beta}[T u(0)]=u_{0}+g(u(t)),
\end{array}\right.
$$

where ${ }^{c} D^{q},{ }^{L} D^{q}$ denote Caputo and Riemann-Liouville fractional order derivatives of order $q \in(0,1)$, respectively, by applying the monotone iterative technique coupled with the method of lower and upper solutions.

However, as far as we know, there have been few applicable results on the existence and uniqueness of solutions to the Hilfer fractional evolution equations by applying the monotone iterative technique and the method of upper and lower solutions. So far we have not seen relevant papers that study Hilfer fractional evolution equations with nonlocal problems by applying the monotone iterative technique and the method of lower and upper solutions. Motivated by these facts, in this work, we use the fixed point theorem combined with monotone iterative technique to discuss the existence of extremal mild solutions for Hilfer fractional evolution equations with nonlocal conditions

$$
\begin{cases}D_{0+}^{v, \mu} u(t)+A u(t)=f(t, u(t), G u(t)), & t \in(0, b] \\ I_{0+}^{(1-v)(1-\mu)} u(0)=u_{0}+\sum_{i=1}^{m} \lambda_{i} u\left(\tau_{i}\right), & \tau_{i} \in(0, b]\end{cases}
$$

where $D_{0+}^{v, \mu}$ denotes the Hilfer fractional derivative of order $\mu$ and type $v$, which will be given in the next section, $0 \leq v \leq 1, \frac{1}{2}<\mu<1$, the state $u(\cdot)$ takes value in a Banach space $E$ with norm $\|\cdot\|$ and $-A: D(A) \subset E \rightarrow E$ is the infinitesimal generator of a $C_{0}$-semigroup $\{T(t)\}_{t \geq 0}$ of uniformly bounded linear operators in $E . J=[0, b](b>0), J^{\prime}=(0, b], f: J^{\prime} \times E \times$ $E \rightarrow E$ are given functions satisfying some assumptions, $u_{0} \in E$ and $\tau_{i}(i=1,2, \ldots, m)$ are prefixed points satisfying $0<\tau_{1} \leq \cdots \leq \tau_{m}<b$, and $\lambda_{i}$ are real numbers. Here the nonlocal condition $I_{0+}^{1-\gamma} u(0)=u_{0}+\sum_{i=1}^{m} \lambda_{i} u\left(\tau_{i}\right)$ can be applied in a physical problem with better effect than the initial condition $I_{0+}^{1-\gamma} u(0)=u_{0}$. The operator $G$ is given by

$$
G u(t)=\int_{0}^{t} K(t, s) u(s) d s
$$

is a Volterra integral operator with integral kernel $K \in C\left(\nabla, \mathbb{R}^{+}\right), \nabla=\{(t, s): 0 \leq s \leq t \leq b\}$. Throughout this paper, we always assume that

$$
K_{0}=\sup _{t \in J} \int_{0}^{t} K(t, s) d s
$$

As far as we know, the nonlocal condition can have a better effect than the initial condition $u(0)=u_{0}$ in physics application. In this article, the nonlocal function $g(u)$ can be given by $g(u)=\sum_{i=1}^{m} \lambda_{i} u\left(\tau_{i}\right)$, we only assume that $\lambda_{i}(i=1,2, \ldots, m)$ satisfy condition $(F 1)$ (see in Sect. 2) without the compactness of nonlocal function. Firstly, we introduce the definition of mild solutions of problem (1.1), and then we prove the existence of extremal mild solutions of problem (1.1) by employing Sadovskii's fixed point theorem. What is more, 
an existence result without using the noncompactness measure condition is obtained in ordered and weakly sequentially complete Banach spaces, which is very useful in application.

Our work is organized as follows: In Sect. 2, we review some essential facts and introduce some notations. In Sect. 3, we state and prove the existence of mild solutions for Hilfer fractional differential system (1.1). Finally, in Sect. 4, an example is given to illustrate the effectiveness of the abstract results.

\section{Preliminaries}

Throughout this paper, by $C(J, E)$ and $C\left(J^{\prime}, E\right)$ we denote the spaces of all continuous functions from $J$ to $E$ and $J^{\prime}$ to $E$, respectively. Let $E$ be an ordered Banach space with the norm $\|\cdot\|$ and partial order $\leq$, whose positive cone $P=\{x \in E: x \geq \theta\}$ is normal with normal constant $N$.

Let $\gamma=v+\mu-v \mu$, then $1-\gamma=(1-v)(1-\mu)$, define $C_{1-\gamma}(J, E)=\left\{u \in C\left(J^{\prime}, E\right): t^{1-\gamma} u(t) \in\right.$ $C(J, E)\}$. Clearly, $C_{1-\gamma}(J, E)$ is a Banach space with the norm $\|u\|_{\gamma}=\sup _{t \in J^{\prime}}\left|t^{1-\gamma} u(t)\right|$. And $C_{1-\gamma}(J, E)$ is also an ordered Banach space with the partial order $\leq$ induced by the positive cone $P^{\prime}=\left\{u \in C_{1-\gamma}(J, E) \mid u(t) \geq \theta, t \in J\right\}$, which is also normal with the same normal constant $N$.

For the convenience of discussion, we recall some definitions and basic results on fractional calculus; for more details, see $[2-4,8,16]$.

Definition 2.1 The Riemann-Liouville fractional integral of order $\alpha$ of a function $f$ : $[0, \infty) \rightarrow R$ is defined as

$$
I_{0+}^{\alpha} f(t)=\frac{1}{\Gamma(\alpha)} \int_{0}^{t}(t-s)^{\alpha-1} f(s) d s, \quad t>0, \alpha>0,
$$

provided the right-hand side is point-wise defined on $[0, \infty)$.

Definition 2.2 The Riemann-Liouville derivative of order $\alpha$ with the lower limit zero for a function $f:[0, \infty) \rightarrow R$ can be written as

$$
D_{0^{+}}^{\alpha} f(t)=\frac{1}{\Gamma(n-\alpha)} \frac{d^{n}}{d t^{n}} \int_{0}^{t} \frac{f(s)}{(t-s)^{\alpha+1-n}} d s, \quad t>0, n-1<\alpha<n .
$$

Definition 2.3 The Caputo fractional derivative of order $\alpha$ for a function $f:[0, \infty) \rightarrow R$ can be written as

$$
{ }^{c} D_{0^{+}}^{\alpha} f(t)=D_{0^{+}}^{\alpha}\left[f(t)-\sum_{k=0}^{n-1} \frac{t^{k}}{k !} f^{(k)}(0)\right], \quad t>0, n-1<\alpha<n,
$$

where $n=[\alpha]+1$ and $[\alpha]$ denotes the integer part of $\alpha$.

Definition 2.4 (Hilfer fractional derivative see [1]) The generalized Riemann-Liouville fractional derivative of order $0 \leq v \leq 1$ and $0<\mu<1$ with lower limit $a$ is defined as

$$
D_{a^{+}}^{\nu, \mu} f(t)=I_{a^{+}}^{\nu(1-\mu)} \frac{d}{d t} I_{a+}^{(1-v)(1-\mu)} f(t)
$$

for functions such that the expression on the right-hand side exists. 


\section{Remark 2.1}

(i) If $v=0,0<\mu<1$, and $a=0$, the Hilfer fractional derivative corresponds to the classical Riemann-Liouville fractional derivative

$$
D_{0+}^{0, \mu} f(t)=\frac{d}{d t} I_{0+}^{1-\mu} f(t)=D_{0+}^{\mu} f(t)
$$

(ii) If $v=1,0<\mu<1$, and $a=0$, the Hilfer fractional derivative corresponds to the classical Caputo fractional derivative

$$
D_{0+}^{1, \mu} f(t)=I_{0+}^{1-\mu} \frac{d}{d t} f(t)={ }^{c} D_{0^{+}}^{\mu} f(t) .
$$

Remark 2.2 The Hilfer fractional derivative is considered as an interpolator between the Riemann-Liouville and Caputo derivatives.

Remark 2.3 For $0<\mu<1$, the Laplace transformation of Hilfer fractional derivatives is given by

$$
\mathcal{L}\left[D_{0+}^{\mu, v} f(x)\right](\lambda)=\lambda^{\mu} \mathcal{L}[f(x)](\lambda)-\lambda^{\nu(\mu-1)}\left(I_{0+}^{(1-v)(1-\mu)} f\right)(0+),
$$

where $\left(I_{0+}^{(1-v)(1-\mu)} f\right)(0+)$ is the Riemann-Liouville fractional integral of order $(1-v)(1-\mu)$ in the limits as $t \rightarrow 0+$, and

$$
\mathcal{L}[f(x)](\lambda)=\int_{0}^{\infty} e^{-\lambda x} f(x) d x
$$

The symbol $\alpha(\cdot)$ is the Kuratowski noncompactness measure defined on a bounded subset $\Omega$ of $E$. For any $\Omega \subset C(J, E)$ and $t \in J$, set $\Omega(t)=\{u(t): u \in B\} \subset E$. If $B$ is bounded in $C(J, E)$, then $\Omega(t)$ is bounded in $E$, and $\alpha(\Omega(t)) \leq \alpha(\Omega)$. As is well known, the Kuratowski measure of noncompactness has the following properties.

Lemma 2.1 ([17]) Let $B \subset C(J, E)$ be bounded and equicontinuous, then $\overline{c o} B \subset C(J, E)$ is also bounded and equicontinuous.

Lemma 2.2 ([18]) Let $E$ be a Banach space, and let $D \subset E$ be bounded. Then there exists a countable set $D_{0} \subset D$ such that $\alpha(D) \leq 2 \alpha\left(D_{0}\right)$.

Lemma 2.3 ([19]) Let E be a Banach space, and let $\Omega \subset C(J, E)$ be equicontinuous and bounded, then $\alpha(\Omega(t))$ is continuous on $J$, and $\alpha(\Omega)=\max _{t \in J} \alpha(\Omega(t))$.

Lemma 2.4 ([20]) Let $\Omega=\left\{u_{n}\right\}_{n=1}^{\infty} \subset C(J, E)$ be a bounded and countable set, and there exists a function $m \in L^{1}\left(J, R^{+}\right)$such that, for every $n \in N$,

$$
\left\|u_{n}(t)\right\| \leq m(t), \quad \text { a.e. } t \in J \text {. }
$$

Then $\alpha(\Omega(t))$ is Lebesgue integral on J, and

$$
\alpha\left(\left\{\int_{J} u_{n}(t) d t: n \in \mathbb{N}\right\}\right) \leq 2 \int_{J} \alpha(\Omega(t)) d t
$$


Based on Lemma 2.12 in paper [2], we give the following the lemma.

Lemma 2.5 Assume that $-A$ is the infinitesimal generator of a $C_{0}$-semigroup $\{T(t)\}_{t \geq 0}$ of uniformly bounded linear operators in $E$. Iff $\in C_{1-\gamma}(J, E)$ for any $u \in C_{1-\gamma}(J, E)$, a function $u$ is a solution of the equation

$$
\left\{\begin{array}{l}
D_{0+}^{\nu, \mu} u(t)+A u(t)=f(t, u(t), G u(t)), \quad t \in J^{\prime} \\
I_{0+}^{1-\gamma} u(0)=u_{0}
\end{array}\right.
$$

if and only if $u$ satisfies the following integral equation:

$$
u(t)=S_{v, \mu}(t) u_{0}+\int_{0}^{t} K_{\mu}(t-s) f(s, u(s), G u(s)) d s
$$

where

$$
S_{\nu, \mu}(t)=I_{0+}^{\nu(1-\mu)} K_{\mu}(t), \quad K_{\mu}(t)=\mu \int_{0}^{\infty} \sigma t^{\mu-1} \xi_{\mu}(\sigma) T\left(t^{\mu} \sigma\right) u_{0} d \sigma
$$

the function $\xi_{\mu}$ is the function of Wright type

$$
\xi_{\mu}(\sigma)=\frac{1}{\pi \mu} \sum_{n=1}^{\infty}(-\sigma)^{n-1} \frac{\Gamma(n \mu+1)}{n !} \sin (n \pi \mu), \quad \sigma \in(0, \infty) .
$$

Lemma 2.6 ([2]) Assume that A generates a $C_{0}$-semigroup $\{T(t)\}_{t \geq 0}$ of uniformly bounded linear operators in $E$ and $T(t)$ is continuous in the uniform operator topology for $t>0$. That is, there exists $M \geq 1$ such that $\sup _{t \in[0,+\infty)}|T(t)| \leq M$. Then the operators $S_{v, \mu}(t)$ and $K_{\mu}(t)$ have the following properties.

(i) For any fixed $t \geq 0,\left\{S_{v, \mu}(t)\right\}_{t>0}$ and $\left\{K_{\mu}(t)\right\}_{t>0}$ are linear operators, and for any $u \in E$,

$$
\left\|S_{v, \mu}(t) u\right\| \leq \frac{M t^{\gamma-1}}{\Gamma(\gamma)}\|u\|, \quad\left\|K_{\mu}(t) u\right\| \leq \frac{M t^{\mu-1}}{\Gamma(\mu)}\|u\| .
$$

(ii) The operators $S_{v, \mu}(t)$ and $K_{\mu}(t)$ are strongly continuous for all $t \geq 0$.

(iii) If $T(t)(t \geq 0)$ is an equicontinuous semigroup, then $S_{v, \mu}(t)$ and $K_{\mu}(t)$ are equicontinuous in $E$ for $t>0$.

In view of [2], from Lemma 2.6, we adopt the following definition of mild solution of system (2.2).

Definition 2.5 A function $u \in C_{1-\gamma}(J, E)$ is said to be a mild solution of (2.2) if $u_{0} \in E$, the integral equation

$$
u(t)=S_{v, \mu}(t) u_{0}+\int_{0}^{t} K_{\mu}(t-s) f(s, u(s), G u(s)) d s
$$

is satisfied for all $t \in J^{\prime}$. 
Next, we present a useful lemma that plays an important role in our main results.

Lemma 2.7 Suppose that $A$ is the infinitesimal generator of a $C_{0}$-semigroup $\{T(t)\}_{t \geq 0}$ of uniformly bounded linear operators in $E$ for $0 \leq v \leq 1,0<\mu<1$, then

$$
D_{0+}^{v, \mu}\left(S_{v, \mu}(t) u_{0}\right)=A\left(S_{v, \mu}(t) u_{0}\right)
$$

and

$$
\begin{aligned}
D_{0+}^{v, \mu} & \left(\int_{0}^{t} K_{\mu}(t-s) f(s, u(s), G u(s)) d s\right) \\
\quad= & A \int_{0}^{t} K_{\mu}(t-s) f(s, u(s), G u(s)) d s+f(t, u(t), G u(t)) .
\end{aligned}
$$

Proof Let $\lambda>0$, we consider the one-sided stable probability density as follows:

$$
\varpi_{\mu}(\sigma)=\frac{1}{\pi} \sum_{n=1}^{\infty}(-1)^{n-1} \sigma^{-\mu n-1} \frac{\Gamma(n \mu+1)}{n !} \sin (n \pi \mu), \quad \sigma \in(0, \infty),
$$

whose Laplace transform is given by

$$
\int_{0}^{\infty} e^{-\lambda \sigma} \varpi_{\mu}(\sigma) d \sigma=e^{-\lambda^{\mu}}, \quad \mu \in(0,1)
$$

Then, using (2.5), we have

$$
\begin{aligned}
\left(\lambda^{\mu} I-A\right)^{-1} u & =\int_{0}^{\infty} e^{-\lambda^{\mu} s} T(s) u_{0} d s=\int_{0}^{\infty} \mu t^{\mu-1} e^{-(\lambda t)^{\mu}} T\left(t^{\mu}\right) u d t \\
& =\int_{0}^{\infty} \int_{0}^{\infty} e^{-(\lambda t \sigma)} \mu t^{\mu-1} \varpi_{\mu}(\sigma) W\left(t^{\mu}\right) u d \sigma d t \\
& =\mu \int_{0}^{\infty} \int_{0}^{\infty} e^{-\lambda \theta} \frac{\theta^{\mu-1}}{\sigma^{\mu}} \varpi_{\mu}(\sigma) T\left(\frac{\theta^{\mu}}{\sigma^{\mu}}\right) u d \theta d \sigma \\
& =\int_{0}^{\infty} e^{-\lambda \tau}\left[\mu \int_{0}^{\infty} \frac{\tau^{\mu-1}}{\sigma^{\mu}} \varpi_{\mu}(\sigma) T\left(\frac{\tau^{\mu}}{\sigma^{\mu}}\right) u d \sigma\right] d \tau \\
& =\int_{0}^{\infty} e^{-\lambda t}\left[\mu \int_{0}^{\infty} \frac{t^{\mu-1}}{\sigma^{\mu}} \varpi_{\mu}(\sigma) T\left(\frac{t^{\mu}}{\sigma^{\mu}}\right) u d \sigma\right] d t \\
& =\int_{0}^{\infty} e^{-\lambda t}\left[\mu \int_{0}^{\infty} \sigma t^{\mu-1} \xi_{\mu}(\sigma) T\left(t^{\mu} \sigma\right) u d \sigma\right] d t \\
& =\int_{0}^{\infty} e^{-\lambda t} K_{\mu}(t) u d t,
\end{aligned}
$$

where $\xi_{\mu}$ is a probability density function defined on $(0, \infty)$ such that

$$
\xi_{\mu}(\sigma)=\frac{1}{\mu} \sigma^{-1-\frac{1}{\mu}} \varpi_{\mu}\left(\sigma^{-\frac{1}{\mu}}\right) \geq 0
$$


Since the Laplace inverse transform of $\lambda^{\nu(\mu-1)}$ is

$$
\mathcal{L}^{-1}\left(\lambda^{\nu(\mu-1)}\right)= \begin{cases}\frac{t^{\nu(1-\mu)-1}}{\Gamma(v(1-\mu))}, & 0<v \leq 1 \\ \delta(t), & v=0\end{cases}
$$

where $\delta(t)$ is the delta function.

From (2.6), (2.7), and the Laplace transform, it is obvious to see that

$$
\begin{aligned}
\mathcal{L}\left(S_{v, \mu}(t) u_{0}\right) & =\mathcal{L}\left(I_{0+}^{v(1-\mu)} K_{\mu}(t) u_{0}\right) \\
& =\mathcal{L}\left(\frac{t^{\nu(1-\mu)-1}}{\Gamma(\nu(1-\mu))} * K_{\mu}(t) u_{0}\right) \\
& =\mathcal{L}\left(\mathcal{L}^{-1}\left(\lambda^{\nu(\mu-1)}\right) * K_{\mu}(t) u_{0}\right) \\
& =\lambda^{\nu(\mu-1)}\left(\lambda^{\mu} I-A\right)^{-1} u_{0},
\end{aligned}
$$

where $*$ denotes the convolution of functions. By Remark 2.2, we obtain

$$
\begin{aligned}
\mathcal{L}\left(D_{0+}^{v, \mu}\left[S_{v, \mu}(t) u_{0}\right]\right) & =\lambda^{\mu} \mathcal{L}\left(S_{v, \mu}(t) u_{0}\right)-\lambda^{v(\mu-1)} u_{0} \\
& =\lambda^{\mu}\left[\lambda^{v(\mu-1)}\left(\lambda^{\mu} I-A\right)^{-1}\right] u_{0}-\lambda^{v(\mu-1)} u_{0} \\
& =\lambda^{\nu(\mu-1)}\left(\lambda^{\mu} I-A\right)^{-1}\left[\lambda^{\mu}-\left(\lambda^{\mu}-A\right)\right] u_{0} \\
& =\lambda^{v(\mu-1)}\left(\lambda^{\mu} I-A\right)^{-1}\left[\lambda^{\mu}-\lambda^{\mu}+A\right] u_{0} \\
& =\lambda^{v(\mu-1)}\left(\lambda^{\mu} I-A\right)^{-1} A u_{0} \\
& =A \lambda^{v(\mu-1)}\left(\lambda^{\mu} I-A\right)^{-1} u_{0} .
\end{aligned}
$$

Combining (2.8) and (2.9) yields

$$
D_{0+}^{v, \mu}\left[S_{v, \mu}(t) u_{0}\right]=A\left[S_{v, \mu}(t) u_{0}\right]
$$

Similarly, we have

$$
\mathcal{L}\left(\int_{0}^{t} K_{\mu}(t-s) f(s, u(s), G u(s)) d s\right)=\mathcal{L}\left(K_{\mu}(t)\right) \cdot \mathcal{L}(f(t, u(t), G u(t)))
$$

and

$$
\begin{aligned}
\mathcal{L} & \left(D_{0+}^{v, \mu}\left[\int_{0}^{t} K_{\mu}(t-s) f(s, u(s), G u(s)) d s\right]\right) \\
& =\lambda^{\mu} \mathcal{L}\left(\int_{0}^{t} K_{\mu}(t-s) f(s, u(s), G u(s)) d s\right)-\lambda^{\nu(\mu-1)} \cdot 0 \\
& =\lambda^{\mu} \mathcal{L}\left(K_{\mu}(t)\right) \cdot \mathcal{L}(f(t, u(t), G u(t))) \\
& =\lambda^{\mu}\left(\lambda^{\mu} I-A\right)^{-1} \cdot \mathcal{L}(f(t, u(t), G u(t))) \\
& =\left(\lambda^{\mu} I-A+A\right)\left(\lambda^{\mu} I-A\right)^{-1} \cdot \mathcal{L}(f(t, u(t), G u(t))) \\
& =A\left(\lambda^{\mu} I-A\right)^{-1} \cdot \mathcal{L}(f(t, u(t), G u(t)))+\mathcal{L}(f(t, u(t), G u(t))) .
\end{aligned}
$$


Thus, it follows from (2.10) and (2.11) that

$$
\begin{aligned}
& D_{0+}^{v, \mu}\left[\int_{0}^{t} K_{\mu}(t-s) f(s, u(s), G u(s)) d s\right] \\
& \quad=A \int_{0}^{t} K_{\mu}(t-s) f(s, u(s), G u(s)) d s+f(t, u(t), G u(t)),
\end{aligned}
$$

which completes the proof of Lemma 2.7.

For the convenience of discussion, we assume the following:

(H0) Assume that $A$ generates a $C_{0}$-semigroup $\{T(t)\}_{t \geq 0}$ of uniformly bounded linear operators in $E$ and $T(t)$ is continuous in the uniform operator topology for $t>0$.

That is, there exists $M \geq 1$ such that $\sup _{t \in[0,+\infty)}\|T(t)\| \leq M$.

(H1) $\lambda_{i}>0(i=1,2, \ldots, m)$ and $\sum_{i=1}^{m} \lambda_{i}<\frac{\Gamma(\gamma)}{M b^{\gamma-1}}$.

In view of [14] and [11], we present the following lemma.

Lemma 2.8 Assume that (H0) and (H1) hold. For any $u \in C_{1-\gamma}(J)$ such that $f(\cdot, u, G u) \in$ $C_{1-\gamma}(J)$, problem (1.1) has a unique mild solution $u \in C_{1-\gamma}(J)$ given by

$$
\begin{aligned}
u(t)= & S_{v, \mu}(t) \bar{\Theta} u_{0}+\sum_{i=1}^{m} \lambda_{i} S_{v, \mu}(t) \bar{\Theta} \int_{0}^{\tau_{i}} K_{\mu}\left(\tau_{i}-s\right) f(s, u(s), G u(s)) d s \\
& +\int_{0}^{t} K_{\mu}(t-s) f(s, u(s), G u(s)) d s
\end{aligned}
$$

where $\bar{\Theta}=\left[I-\sum_{i=1}^{m} \lambda_{i} S_{v, \mu}\left(\tau_{i}\right)\right]^{-1}$.

Proof By assumption (H0), we have

$$
\left\|\sum_{i=1}^{m} \lambda_{i} S_{v, \mu}(t)\right\| \leq \sum_{i=1}^{m}\left|\lambda_{i}\right| \cdot\left\|S_{\nu, \mu}(t)\right\| \leq \sum_{i=1}^{m}\left|\lambda_{i}\right| \frac{M b^{\gamma-1}}{\Gamma(\gamma)}<1
$$

By operator spectrum theorem, the operator $\left.\bar{\Theta}:=\left(I-\sum_{i=1}^{m} \lambda_{i} S_{v, \mu}\left(\tau_{i}\right)\right)\right)^{-1}$ exists and is bounded. Furthermore, by Neumann's expression, we obtain

$$
\|\bar{\Theta}\| \leq \sum_{i=0}^{\infty}\left\|\sum_{i=1}^{m} \lambda_{i} S_{v, \mu}\left(\tau_{i}\right)\right\|^{n}=\frac{1}{1-\left\|\sum_{i=1}^{m} \lambda_{i} S_{v, \mu}\left(\tau_{i}\right)\right\|} \leq \frac{1}{1-\frac{M b^{\gamma-1}}{\Gamma(\gamma)} \sum_{i=1}^{m} \lambda_{i}} .
$$

According to Definition 2.5, a solution of system (2.2) can be expressed by

$$
u(t)=S_{v, \mu}(t) I_{0+}^{1-\gamma} u(0)+\int_{0}^{t} K_{\mu}(t-s) f(s, u(s), G u(s)) d s
$$

Next, we substitute $t=\tau_{i}$ into (2.13), and by applying $\lambda_{i}$ to both sides of (2.13), we have

$$
\lambda_{i} u\left(\tau_{i}\right)=\lambda_{i} S_{\nu, \mu}\left(\tau_{i}\right) I_{0+}^{1-\gamma} u(0)+\lambda_{i} \int_{0}^{\tau_{i}} K_{\mu}\left(\tau_{i}-s\right) f(s, u(s), G u(s)) d s .
$$


Thus, we have

$$
\begin{aligned}
I_{0+}^{1-\gamma} u(0) & =u_{0}+\sum_{i=1}^{m} \lambda_{i} u\left(\tau_{i}\right) \\
& =u_{0}+\sum_{i=1}^{m} \lambda_{i} S_{v, \mu}\left(\tau_{i}\right) I_{0+}^{1-\gamma} u(0)+\sum_{i=1}^{m} \lambda_{i} \int_{0}^{\tau_{i}} K_{\mu}\left(\tau_{i}-s\right) f(s, u(s), G u(s)) d s \\
& =u_{0}+\sum_{i=1}^{m} \lambda_{i} S_{v, \mu}\left(\tau_{i}\right) I_{0+}^{1-\gamma} u(0)+\sum_{i=1}^{m} \lambda_{i} \int_{0}^{\tau_{i}} K_{\mu}\left(\tau_{i}-s\right) f(s, u(s), G u(s)) d s .
\end{aligned}
$$

Since $I-\sum_{i=1}^{m} \lambda_{i} S_{v, \mu}\left(\tau_{i}\right)$ has a bounded inverse operator $\bar{\Theta}$, it implies

$$
\begin{aligned}
I_{0+}^{1-\gamma} & u(0) \\
= & {\left[I-\sum_{i=1}^{m} \lambda_{i} S_{v, \mu}\left(\tau_{i}\right)\right]^{-1}\left(u_{0}+\sum_{i=1}^{m} \lambda_{i} \int_{0}^{\tau_{i}} K_{\mu}\left(\tau_{i}-s\right) f(s, u(s), G u(s)) d s\right) } \\
= & \bar{\Theta} u_{0}+\sum_{i=1}^{m} \lambda_{i} \int_{0}^{\tau_{i}} \bar{\Theta} K_{\mu}\left(\tau_{i}-s\right) f(s, u(s), G u(s)) d s .
\end{aligned}
$$

Submitting (2.1) to (2.14), we obtain that (2.13). It implies that $u$ is also a solution of the integral of Eq. (2.13) when $u$ is a solution of system (2.12).

The necessity has been proved. Next, we will prove its sufficiency. Applying $I_{0+}^{1-\gamma}$ to both sides of (2.12), and by Lemma 2.7, we have

$$
\begin{aligned}
I_{0+}^{1-\gamma} u(t)= & I_{0+}^{1-\gamma}\left(S_{v, \mu}(t) \bar{\Theta} u_{0}+\sum_{i=1}^{m} \lambda_{i} S_{v, \mu}(t) \bar{\Theta} \int_{0}^{\tau_{i}} K_{\mu}\left(\tau_{i}-s\right) f(s, u(s), G u(s)) d s\right. \\
& \left.+\int_{0}^{t} K_{\mu}(t-s) f(s, u(s), G u(s)) d s\right) .
\end{aligned}
$$

Therefore, we have

$$
\begin{aligned}
& \lim _{t \rightarrow 0} I_{0+}^{1-\gamma} u(t) \\
& \quad=\lim _{t \rightarrow 0} I_{0+}^{1-\gamma} S_{v, \mu}(t) \bar{\Theta} u_{0}+\sum_{i=1}^{m} \lambda_{i} \lim _{t \rightarrow 0} I_{0+}^{1-\gamma} S_{v, \mu}(t) \bar{\Theta} \int_{0}^{\tau_{i}} K_{\mu}\left(\tau_{i}-s\right) f(s, u(s), G u(s)) d s \\
& \quad=I_{0+}^{1-\gamma}\left(\lim _{t \rightarrow 0} S_{\nu, \mu}(t)\left(\bar{\Theta} u_{0}\right)+I_{0+}^{1-\gamma} \lim _{t \rightarrow 0} S_{\nu, \mu}(t) \sum_{i=1}^{m} \lambda_{i} \bar{\Theta} \int_{0}^{\tau_{i}} K_{\mu}\left(\tau_{i}-s\right) f(s, u(s), G u(s)) d s\right. \\
& \quad=I_{0+}^{1-\gamma}\left(\frac{\bar{\Theta} u_{0}}{\Gamma(\gamma)} t^{\gamma-1}\right)+I_{0+}^{1-\gamma}\left(\frac{\sum_{i=1}^{m} \lambda_{i} \bar{\Theta} \int_{0}^{\tau_{i}} K_{\mu}\left(\tau_{i}-s\right) f(s, u(s), G u(s)) d s}{\Gamma(\gamma)} t^{\gamma-1}\right) \\
& \quad=\bar{\Theta} u_{0}+\sum_{i=1}^{m} \lambda_{i} \bar{\Theta} \int_{0}^{\tau_{i}} K_{\mu}\left(\tau_{i}-s\right) f(s, u(s), G u(s)) d s
\end{aligned}
$$


Substituting $t=\tau_{i}$ into (2.12), we have

$$
\begin{aligned}
u\left(\tau_{i}\right)= & S_{\nu, \mu}\left(\tau_{i}\right) \bar{\Theta} u_{0}+\sum_{i=1}^{m} \lambda_{i} S_{v, \mu}\left(\tau_{i}\right) \bar{\Theta} \int_{0}^{\tau_{i}} K_{\mu}\left(\tau_{i}-s\right) f(s, u(s), G u(s)) d s \\
& +\int_{0}^{\tau_{i}} K_{\mu}\left(\tau_{i}-s\right) f(s, u(s), G u(s)) d s .
\end{aligned}
$$

Then we obtain

$$
\begin{aligned}
u_{0}+ & \sum_{i=1}^{m} \lambda_{i} u\left(\tau_{i}\right) \\
= & u_{0}+\sum_{i=1}^{m} \lambda_{i} S_{v, \mu}\left(\tau_{i}\right) \bar{\Theta} u_{0}+\sum_{i=1}^{m} \lambda_{i} \sum_{i=1}^{m} \lambda_{i} S_{v, \mu}\left(\tau_{i}\right) \bar{\Theta} \int_{0}^{\tau_{i}} K_{\mu}\left(\tau_{i}-s\right) f(s, u(s), G u(s)) d s \\
& +\sum_{i=1}^{m} \lambda_{i} \int_{0}^{\tau_{i}} K_{\mu}\left(\tau_{i}-s\right) f(s, u(s), G u(s)) d s \\
= & \left(I+\sum_{i=1}^{m} \lambda_{i} S_{v, \mu}\left(\tau_{i}\right) \bar{\Theta}\right)\left(u_{0}+\sum_{i=1}^{m} \lambda_{i} \int_{0}^{\tau_{i}} K_{\mu}\left(\tau_{i}-s\right) f(s, u(s), G u(s)) d s\right) \\
= & \left(\bar{\Theta}-1+\sum_{i=1}^{m} \lambda_{i} S_{v, \mu}\left(\tau_{i}\right)\right)\left(\bar{\Theta} u_{0}+\sum_{i=1}^{m} \lambda_{i} \bar{\Theta} \int_{0}^{\tau_{i}} K_{\mu}\left(\tau_{i}-s\right) f(s, u(s), G u(s)) d s\right) \\
= & \bar{\Theta} u_{0}+\sum_{i=1}^{m} \lambda_{i} \bar{\Theta} \int_{0}^{\tau_{i}} K_{\mu}\left(\tau_{i}-s\right) f(s, u(s), G u(s)) d s .
\end{aligned}
$$

It follows from (2.16) and (2.17) that $I_{0+}^{1-\gamma} u(0)=u_{0}+\sum_{i=1}^{m} \lambda_{i} u\left(\tau_{i}\right)$.

Next, by using $D_{0+}^{v, \mu}$ to both sides of (2.12) and Lemma 2.9, we have

$$
\begin{aligned}
& D_{0+}^{v, \mu} u(t)=D_{0+}^{v, \mu}\left[S_{v, \mu}(t) \bar{\Theta} u_{0}+\sum_{i=1}^{m} \lambda_{i} S_{v, \mu}(t) \bar{\Theta} \int_{0}^{\tau_{i}} K_{\mu}\left(\tau_{i}-s\right) f(s, u(s), G u(s)) d s\right. \\
& \left.+\int_{0}^{t} K_{\mu}(t-s) f(s, u(s), G u(s)) d s\right] \\
& =D_{0+}^{v, \mu}\left[S_{v, \mu}(t) \bar{\Theta} u_{0}+\sum_{i=1}^{m} \lambda_{i} S_{v, \mu}(t) \bar{\Theta} \int_{0}^{\tau_{i}} K_{\mu}\left(\tau_{i}-s\right) f(s, u(s), G u(s)) d s\right] \\
& +D_{0+}^{v, \mu}\left[\int_{0}^{t} K_{\mu}(t-s) f(s, u(s), G u(s)) d s\right] \\
& =\left[\bar{\Theta} u_{0}+\sum_{i=1}^{m} \lambda_{i} \bar{\Theta} \int_{0}^{\tau_{i}} K_{\mu}\left(\tau_{i}-s\right) f(s, u(s), G u(s)) d s\right] D_{0+}^{v, \mu}\left[S_{v, \mu}(t)\right] \\
& +D_{0+}^{v, \mu}\left[\int_{0}^{t} K_{\mu}(t-s) f(s, u(s), G u(s))\right] \\
& =\left[\bar{\Theta} u_{0}+\sum_{i=1}^{m} \lambda_{i} \bar{\Theta} \int_{0}^{\tau_{i}} K_{\mu}\left(\tau_{i}-s\right) f(s, u(s), G u(s)) d s\right] A S_{\nu, \mu}(t) \\
& +A \int_{0}^{t} K_{\mu}(t-s) f(s, u(s), G u(s)) d s+f(t, u(t), G u(t))
\end{aligned}
$$




$$
\begin{aligned}
= & A\left(S_{v, \mu}(t) \bar{\Theta} u_{0}+\sum_{i=1}^{m} \lambda_{i} S_{v, \mu}(t) \bar{\Theta} \int_{0}^{\tau_{i}} K_{\mu}\left(\tau_{i}-s\right) f(s, u(s), G u(s)) d s\right. \\
& \left.+\int_{0}^{t} K_{\mu}(t-s) f(s, u(s), G u(s)) d s\right)+f(t, u(t), G u(t)) \\
= & A u(t)+f(t, u(t), G u(t)) .
\end{aligned}
$$

Hence,

$$
D_{0+}^{v, \mu} u(t)=A u(t)+f(s, u(t), G u(t)) .
$$

This proof is completed.

From Lemma 2.8, we adopt the following definition of a mild solution of problem (1.1).

Definition 2.6 A function $u \in C_{1-\gamma}(J, E)$ is said to be a mild solution of problem (1.1) if it satisfies the operator equation

$$
\begin{aligned}
u(t)= & S_{v, \mu}(t) \bar{\Theta} u_{0}+\sum_{i=1}^{m} \lambda_{i} S_{v, \mu}(t) \bar{\Theta} \int_{0}^{\tau_{i}} K_{\mu}\left(\tau_{i}-s\right) f(s, u(s), G u(s)) d s \\
& +\int_{0}^{t} K_{\mu}(t-s) f(s, u(s), G u(s)) d s, \quad t \in J^{\prime}
\end{aligned}
$$

where the operators $S_{v, \mu}(t)$ and $K_{\mu}(t)$ are given by (2.3).

Definition 2.7 A $C_{0}$-semigroup $\{T(t)\}_{t \geq 0}$ in $E$ is said to be positive if the order inequality $T(t) x \geq \theta$ holds for each $x \geq \theta, x \in E$, and $t \geq 0$.

Remark 2.4 For any $C \geq 0,-(A+C I)$ also generates a $C_{0}$-semigroup $S(t)=e^{-C t} T(t)(t \geq 0)$ on $E$. And $S(t)(t \geq 0)$ is a positive $C_{0}$-semigroup if $T(t)(t \geq 0)$ is a positive $C_{0}$-semigroup. For details, see $[18,21]$.

For $u \in E$, we define two families $\left\{S_{v, \mu}^{*}(t)\right\}_{t \geq}$ and $\left\{K_{\mu}^{*}(t)\right\}_{t \geq 0}$ of operators by

$$
S_{v, \mu}^{*}(t) u=I_{0+}^{v(1-\mu)} K_{\mu}^{*}(t) u, \quad K_{\mu}^{*}(t) u=\mu \int_{0}^{\infty} \sigma t^{\mu-1} \xi_{\mu}(\sigma) S\left(t^{\mu} \sigma\right) u d \sigma
$$

where $\xi_{\mu}(\sigma)$ is given by (2.3).

Since $T(t)(t \geq 0)$ is positive, by Remark 2.4, it is easy to know that $S(t)(t \geq 0)$ is also positive. And by the definition of $\xi_{\mu}(\sigma)$, the operators $S_{v, \mu}^{*}(t)$ and $K_{\mu}^{*}(t)$ are also positive for all $t \geq 0$.

To prove our main result, for any $C>0$, we consider the following system:

$$
\left\{\begin{array}{l}
D_{0+}^{v, \mu} u(t)+(A+C I) u(t)=f(t, u(t), G u(t))+C u(t), \quad t \in(0, b] \\
I_{0+}^{(1-v)(1-\mu)} u(0)=u_{0}+\sum_{i=1}^{m} \lambda_{i} u\left(\tau_{i}\right), \quad \tau_{i} \in(0, b]
\end{array}\right.
$$

First, we assume the following: 
(F0) For any $C \geq 0,-(A+C I)$ also generates a $C_{0}$-semigroup $S(t)=e^{-C t} T(t)(t \geq 0)$ on $E$ and $S(t)$ is continuous in the uniform operator topology for $t>0$. That is, there exists $M^{*} \geq 1$ such that $\sup _{t \in[0,+\infty)}|S(t)| \leq M^{*}$.

(F1) $\lambda_{i}>0(i=1,2, \ldots, m)$ and $\sum_{i=1}^{m} \lambda_{i}<\frac{\Gamma(\gamma)}{M^{*} b^{\gamma-1}}$.

By assumption (F1), we have

$$
\left\|\sum_{i=1}^{m} \lambda_{i} S_{v, \mu}^{*}(t)\right\| \leq \frac{M^{*} b^{\gamma-1}}{\Gamma(\gamma)} \sum_{i=1}^{m} \lambda_{i}<1
$$

By operator spectrum theorem, the operator $\left.I-\sum_{i=1}^{m} \lambda_{i} S_{v, \mu}^{*}\left(\tau_{i}\right)\right)$ has a bounded inverse operator

$$
\left.\Theta:=\left(I-\sum_{i=1}^{m} \lambda_{i} S_{v, \mu}^{*}\left(\tau_{i}\right)\right)\right)^{-1}
$$

Furthermore, by Neumann's expression, $\bar{\Theta}$ can be expressed by

$$
\Theta=\sum_{i=0}^{\infty}\left(\sum_{i=1}^{m} \lambda_{i} S_{v, \mu}^{*}\left(\tau_{i}\right)\right)^{n}
$$

By the positivity of $C_{0}$-semigroup $S(t)(t \geq 0)$, it is easy to know that $S_{v, \mu}^{*}(t)$ is positive, we have

$$
\Theta u=\sum_{i=0}^{\infty}\left(\sum_{i=1}^{m} \lambda_{i} S_{v, \mu}^{*}\left(\tau_{i}\right)\right)^{n} u \geq u \geq \theta, \quad \forall u \geq \theta
$$

So, $\Theta$ is a positive operator, and

$$
\|\Theta\| \leq \sum_{i=0}^{\infty}\left\|\sum_{i=1}^{m} \lambda_{i} S_{v, \mu}^{*}\left(\tau_{i}\right)\right\|^{n}=\frac{1}{1-\left\|\sum_{i=1}^{m} \lambda_{i} S_{v, \mu}^{*}\left(\tau_{i}\right)\right\|} \leq \frac{1}{1-\frac{M^{*} b^{\gamma-1}}{\Gamma(\gamma)} \sum_{i=1}^{m} \lambda_{i}}
$$

In view of Lemma 2.8, we present the following lemma.

Lemma 2.9 Assume that (F0) and (F1) hold. For any $u \in C_{1-\gamma}(J)$ such that $f(\cdot, u, G u) \in$ $C_{1-\gamma}(J)$, problem (2.20) has a unique mild solution $u \in C_{1-\gamma}(J)$ given by

$$
\begin{aligned}
u(t)= & S_{v, \mu}^{*}(t) \bar{\Theta} u_{0}+\sum_{i=1}^{m} \lambda_{i} S_{v, \mu}^{*}(t) \bar{\Theta} \int_{0}^{\tau_{i}} K_{\mu}^{*}\left(\tau_{i}-s\right)[f(s, u(s), G u(s))+C u(s)] d s \\
& +\int_{0}^{t} K_{\mu}^{*}(t-s)[f(s, u(s), G u(s))+C u(s)] d s,
\end{aligned}
$$

where $\bar{\Theta}=\left[I-\sum_{i=1}^{m} \lambda_{i} S_{v, \mu}^{*}\left(\tau_{i}\right)\right]^{-1}$.

From Lemma 2.9 and Definition 2.7, we state the following definition of a mild solution of problem (2.20). 
Definition 2.8 A function $u \in C_{1-\gamma}(J, E)$ is said to be a mild solution of problem (2.20) if, for any $u \in C_{1-\gamma}(J, E)$, the integral equation

$$
\begin{aligned}
u(t)= & S_{v, \mu}^{*}(t) \bar{\Theta} u_{0}+\sum_{i=1}^{m} \lambda_{i} S_{v, \mu}^{*}(t) \bar{\Theta} \int_{0}^{\tau_{i}} K_{\mu}^{*}\left(\tau_{i}-s\right)[f(s, u(s), G u(s))+C u(s)] d s \\
& +\int_{0}^{t} K_{\mu}^{*}(t-s)[f(s, u(s), G u(s))+C u(s)] d s
\end{aligned}
$$

is satisfied.

In the following, we will state some lemmas whose proofs are similar to those of the paper [2]. Here, we omit it.

Lemma 2.10 Under assumption (F0), the operators $S_{v, \mu}^{*}(t)$ and $K_{\mu}^{*}(t)$ have the following properties:

(i) For any fixed $t>0,\left\{K_{\mu}^{*}(t)\right\}_{t>0}$ and $\left\{S_{v, \mu}^{*}(t)\right\}_{t>0}$ are linear operators, and for any $u \in E$,

$$
\left\|K_{\mu}^{*}(t)\right\| \leq \frac{M^{*} t^{\mu-1}}{\Gamma(\mu)}, \quad\left\|S_{v, \mu}^{*}(t)\right\| \leq \frac{M^{*} t^{\gamma-1}}{\Gamma(\gamma)}
$$

(ii) The operators $\left\{K_{\mu}^{*}(t)\right\}_{t>0}$ and $\left\{S_{v, \mu}^{*}(t)\right\}_{t>0}$ are strongly continuous for $t>0$.

(iii) If $S(t)(t \geq 0)$ is an equicontinuous semigroup, then $S_{v, \mu}^{*}(t)$ and $K_{\mu}^{*}(t)$ are equicontinuous in $E$ for $t>0$.

To end this section, we state a fixed point theorem, which plays a major role in the proof of our main results.

Lemma 2.11 (Sadovskii's fixed point theorem) Let D be a convex, closed, and bounded subset of a Banach space $E$ and $Q: D \rightarrow D$ be a condensing map. Then $Q$ has one fixed point in $D$.

Lemma 2.12 ([22]) Let $a \geq 0, \mu>0, c(t)$, and $u(t)$ be the nonnegative locally integrable functions on $0 \leq t<T<+\infty$ such that

$$
u(t) \leq c(t)+a \int_{0}^{t}(t-s)^{\mu-1} u(s) d s
$$

then

$$
u(t) \leq c(t)+\int_{0}^{t}\left[\sum_{n=1}^{\infty} \frac{(a \Gamma(\mu))^{n}}{\Gamma(n \mu)}(t-s)^{n \mu-1} c(s)\right] d s, \quad 0 \leq t<T .
$$

\section{Main results}

In this section, we discuss the existence of extremal mild solutions for problem (1.1).

Definition 3.1 An abstract function $u \in C_{1-\gamma}(J, E)$ is called a solution of problem (1.1) if $u(t)$ satisfies all the equalities of (1.1). 
Definition 3.2 If the function $v_{0} \in C_{1-\gamma}(J, E)$ satisfies

$$
\left\{\begin{array}{l}
D_{0+}^{v, \mu} v_{0}(t)+A v_{0}(t) \leq f\left(t, v_{0}(t), G v_{0}(t)\right), \quad t \in J \\
I_{0+}^{1-\gamma} v_{0}(0) \leq u_{0}+\sum_{i=1}^{m} \lambda_{i} v_{0}\left(\tau_{i}\right)
\end{array}\right.
$$

then $v_{0}$ is said to be a lower solution of problem (1.1). If all the inequalities in (3.1) are reversed, then $v_{0}$ is called an upper solution of problem (1.1).

Theorem 3.1 Assume that $E$ is an ordered Banach space, its positive cone $P$ is normal, and-A generates a positive $C_{0}$-semigroup $\{T(t)\}_{t \geq 0}$ on $E, f \in C(J \times E \times E, E)$, and $u_{0} \in E$. If problem (1.1) has a lower solution $v_{0} \in C_{1-\gamma}(J, E)$ and an upper solution $w_{0} \in C_{1-\gamma}(J, E)$ with $v_{0} \leq w_{0}$. Suppose also that conditions (F0), (F1) and the following conditions are satisfied:

(F2) There exists a constant $C>0$ satisfying

$$
f\left(t, u_{2}, v_{2}\right)-f\left(t, u_{1}, v_{1}\right) \geq-C\left(u_{2}-u_{1}\right)
$$

for $\forall \in J$, and $v_{0}(t) \leq u_{1} \leq u_{2} \leq w_{0}(t), G v_{0}(t) \leq v_{1} \leq v_{2} \leq G w_{0}(t)$.

(F3) There exists a constant $L>0$ satisfying

$$
\alpha\left(\left\{f\left(t, u_{n}, v_{n}\right)\right\}\right) \leq L\left(\alpha\left(\left\{u_{n}\right\}\right)+\alpha\left(\left\{v_{n}\right\}\right)\right)
$$

for $\forall t \in J$, and increasing or decreasing monotonic sequences $\left\{u_{n}\right\} \subset\left[v_{0}(t), w_{0}(t)\right]$ and $\left\{v_{n}\right\} \subset\left[G v_{0}(t), G w_{0}(t)\right]$.

(F4) Let $v_{n}=Q v_{n-1}, w_{n}=Q w_{n-1}, n=1,2, \ldots$, such that the sequences $v_{n}(0)$ and $w_{n}(0)$ are convergent.

Then problem (1.1) has minimal and maximal mild solutions $\underline{u}$ and $\bar{u}$ between $v_{0}$ and $w_{0}$, which can be obtained by using the monotone iterative procedure starting from $v_{0}$ and $w_{0}$ respectively.

Proof Since $C>0$, problem (1.1) can be written as system (2.20). By (2.21), we can define operator $Q:\left[v_{0}, w_{0}\right] \rightarrow C_{1-\gamma}(J, E)$ as follows:

$$
\begin{aligned}
(Q u)(t)= & S_{v, \mu}^{*}(t) \Theta u_{0}+\sum_{i=1}^{m} \lambda_{i} S_{v, \mu}^{*}(t) \Theta \int_{0}^{\tau_{i}} K_{\mu}^{*}\left(\tau_{i}-s\right)[f(s, u(s), G u(s))+C u(s)] d s \\
& +\int_{0}^{t} K_{\mu}^{*}(t-s)[f(s, u(s), G u(s))+C u(s)] d s, \quad t \in J^{\prime} .
\end{aligned}
$$

Since $f$ is continuous, it is easily seen that the map $Q:\left[v_{0}, w_{0}\right] \rightarrow C_{1-\gamma}(J, E)$ is continuous. And by Lemma 2.9, the mild solutions of problem (1.1) are equivalent to the fixed points of the operator $Q$. We will divide the proof in the following steps.

Step 1. We show that $Q:\left[v_{0}, w_{0}\right] \rightarrow C_{1-\gamma}(J, E)$ is an increasing monotone operator. In fact, for $\forall t \in J^{\prime}, v_{0}(t) \leq u \leq v \leq w_{0}$, by assumptions (F2) and (F3), we have

$$
f\left(s, v_{0}(s), G v_{0}(s)\right)+C v_{0}(s) \leq f\left(s, w_{0}(s), G w_{0}(s)\right)+C w_{0}(s) .
$$


So

$$
\begin{aligned}
& \int_{0}^{t} K_{\mu}^{*}(t-s)[f(s, u(s), G u(s))+C u(s)] d s \\
& \quad \leq \int_{0}^{t} K_{\mu}^{*}(t-s)[f(s, v(s), G v(s))+C v(s)] d s .
\end{aligned}
$$

Thus, from (3.2) we have $Q u \leq Q v$.

Step 2. We show that $v_{0} \leq Q v_{0}$ and $Q w_{0} \leq w_{0}$. Let $h(t)=D_{0+}^{v, \mu} v_{0}(t)+A v_{0}(t)+C v_{0}(t)$, $h \in C_{1-\gamma}(J, E)$, and $h(t) \leq f\left(t, v_{0}(t), G v_{0}(t)\right)+C v_{0}(t), t \in J^{\prime}$. By Definitions 2.7 and 3.2, we have

$$
\begin{aligned}
v_{0}(t)= & S_{v, \mu}^{*}(t) v_{0}(0)+\int_{0}^{t} K_{\mu}^{*}(t-s) h(s) d s \\
\leq & S_{v, \mu}^{*}(t) \Theta u_{0}+\sum_{i=1}^{m} \lambda_{i} S_{v, \mu}^{*}(t) \Theta \int_{0}^{\tau_{i}} K_{\mu}^{*}\left(\tau_{i}-s\right)\left[f\left(s, v_{0}(s), G v_{0}(s)\right)+C v_{0}(s)\right] d s \\
& +\int_{0}^{t} K_{\mu}^{*}(t-s)\left[f\left(s, v_{0}(s), G v_{0}(s)\right)+C v_{0}(s)\right] d s \\
= & Q v_{0}(t), \quad t \in J^{\prime} .
\end{aligned}
$$

It implies that $v_{0} \leq Q v_{0}$. Similarly, it can prove that $Q w_{0} \leq w_{0}$. Thus, $Q:\left[v_{0}, w_{0}\right] \rightarrow\left[v_{0}, w_{0}\right]$ is a continuous increasing monotone operator.

Now, we define two sequences $\left\{v_{n}\right\}$ and $\left\{w_{n}\right\}$ in $\left[v_{0}, w_{0}\right]$ by the iterative scheme

$$
v_{n}=Q v_{n-1}, \quad w_{n}=Q w_{n-1}, \quad n=1,2, \ldots
$$

Then, from the monotonicity of $Q$, we have

$$
v_{0} \leq v_{1} \leq v_{2} \leq \cdots \leq v_{n} \leq \cdots \leq w_{n} \leq \cdots \leq w_{2} \leq w_{1} \leq w_{0}
$$

Step 3. We prove that $\left\{v_{n}\right\}$ and $\left\{w_{n}\right\}$ are convergent in $J^{\prime}$.

Let $B=\left\{v_{n}: n \in \mathbb{N}\right\}$ and $B_{0}=\left\{v_{n-1}: n \in \mathbb{N}\right\}$. Then $B=Q\left(B_{0}\right)$. From $B_{0}=B \cup\left\{v_{0}\right\}$ it follows that $\alpha\left(B_{0}(t)\right)=\alpha(B(t))$ for $t \in J^{\prime}$. Let $\varphi(t):=\alpha(B(t)), t \in J^{\prime}$, we will show that $\varphi(t) \equiv 0$ in $J^{\prime}$.

For $t \in J^{\prime}$, by (1.2) and Lemma 2.4, we get

$$
\begin{aligned}
\alpha\left(G\left(B_{0}\right)(t)\right) & =\alpha\left(\left\{\int_{0}^{t} K(t, s) v_{n-1}(s) d s: n \in \mathbb{N}\right\}\right) \\
& \leq 2 K_{0} \int_{0}^{t} \alpha\left(B_{0}(s)\right) d s \\
& =2 K_{0} \int_{0}^{t} \varphi(s) d s,
\end{aligned}
$$

therefore

$$
\int_{0}^{t} \alpha\left(G\left(B_{0}\right)(s)\right) d s \leq 2 b K_{0} \int_{0}^{t} \varphi(s) d s .
$$


For $t \in J^{\prime}$, from (3.2), using Lemma 2.2, assumptions (F3) and (F4), we have

$$
\begin{aligned}
\varphi(t)= & \alpha(B(t))=\alpha\left(Q\left(B_{0}\right)(t)\right) \\
= & \alpha\left(\left\{S_{v, \mu}^{*}(t) \Theta u_{0}\right.\right. \\
& +\sum_{i=1}^{m} \lambda_{i} S_{v, \mu}^{*}(t) \Theta \int_{0}^{\tau_{i}} K_{\mu}^{*}\left(\tau_{i}-s\right)\left[f\left(s, v_{n-1}(s), G v_{n-1}(s)\right)+C v_{n-1}(s)\right] d s \\
& \left.\left.+\int_{0}^{t} K_{\mu}^{*}(t-s)\left[f\left(s, v_{n-1}(s), G v_{n-1}(s)\right)+C v_{n-1}(s)\right] d s\right\}\right) \\
\leq & \frac{M^{*} b^{\gamma-1}}{\Gamma(\gamma)} \\
& \times \alpha\left(\left\{\Theta u_{0}+\sum_{i=1}^{m} \lambda_{i} \Theta \int_{0}^{\tau_{i}} K_{\mu}^{*}\left(\tau_{i}-s\right)\left[f\left(s, v_{n-1}(s), G v_{n-1}(s)\right)+C v_{n-1}(s)\right] d s\right\}\right) \\
& +\frac{2 M^{*} b^{\mu-1}}{\Gamma(\mu)} \int_{0}^{t} \alpha\left(\left\{f\left(s, v_{n-1}(s), G v_{n-1}(s)\right)+C v_{n-1}(s)\right\}\right) d s \\
\leq & \frac{M^{*} b^{\gamma-1}}{\Gamma(\gamma)} \alpha\left(\left\{v_{n}(0)\right\}\right)+\frac{2 M^{*} b^{\mu-1}\left(L+2 b L K_{0}+C\right)}{\Gamma(\mu)} \int_{0}^{t} \alpha\left(B_{0}(s)\right) d s \\
\leq & \frac{2 M^{*} b^{\mu-1}\left(L+2 b L K_{0}+C\right)}{\Gamma(\mu)} \int_{0}^{t} \varphi(s) d s .
\end{aligned}
$$

Hence, by Lemma 2.12, $\varphi(t) \equiv 0$ in $J$. So, for any $t \in J,\left\{v_{n}(t)\right\}$ is precompact and $\left\{v_{n}(t)\right\}$ has a convergent subsequence. And by the monotonicity of (3.3), we prove that $\left\{v_{n}(t)\right\}$ itself is convergent, i.e., $\lim _{n \rightarrow \infty} v_{n}(t)=\underline{u}(t), t \in J$. Similarly, $\lim _{n \rightarrow \infty} w_{n}(t)=\bar{u}(t), t \in J$.

Evidently, $\left\{v_{n}(t)\right\} \in C_{1-\gamma}(J, E)$, so $\underline{u}(t)$ is bounded integrable on $J$. For any $t \in J$,

$$
\begin{aligned}
v_{n}(t)= & Q\left(v_{n-1}\right) \\
= & S_{v, \mu}^{*}(t) \Theta u_{0}+\sum_{i=1}^{m} \lambda_{i} S_{v, \mu}^{*}(t) \Theta \int_{0}^{\tau_{i}} K_{\mu}^{*}\left(\tau_{i}-s\right)\left[f\left(s, v_{n-1}(s), G v_{n-1}(s)\right)+C v_{n-1}(s)\right] d s \\
& +\int_{0}^{t} K_{\mu}^{*}(t-s)\left[f\left(s, v_{n-1}(s), G v_{n-1}(s)\right)+C v_{n-1}(s)\right] d s
\end{aligned}
$$

If $n \rightarrow \infty$ in (3.5), by the Lebesgue dominated convergence theorem, we obtain

$$
\begin{aligned}
\underline{u}(t)= & Q(\underline{u}(t)) \\
= & S_{v, \mu}^{*}(t) \Theta u_{0}+\sum_{i=1}^{m} \lambda_{i} S_{v, \mu}^{*}(t) \Theta \int_{0}^{\tau_{i}} K_{\mu}^{*}\left(\tau_{i}-s\right)[f(s, \underline{u}(s), G \underline{u}(s))+C \underline{u}(s)] d s \\
& +\int_{0}^{t} K_{\mu}^{*}(t-s)[f(s, \underline{u}(s), G \underline{u}(s))+C \underline{u}(s)] d s .
\end{aligned}
$$

Thus, we have $\underline{u}(t) \in C_{1-\gamma}(J, E)$ and $\underline{u}=Q \underline{u}$. In a similar way, we can prove that there exists $\bar{u}(t) \in C_{1-\gamma}(J, E)$ such that $\bar{u}=Q \bar{u}$. Combining this with the monotonicity of (3.4), we see that $v_{0} \leq \underline{u} \leq \bar{u} \leq w_{0}$, which implies that $\underline{u}$ and $\bar{u}$ are the minimal and maximal mild solutions of problem (1.1) in $\left[v_{0}, w_{0}\right]$. 
Remark 3.1 If we replace positive cone $P$ is normal by positive cone $P$ is regular, then the conclusion in Theorem 3.1 is also valid. For more details, see [14].

As a supplement to Theorem 3.1, we further discuss the existence of mild solutions for problem (1.1) in a weakly sequentially complete Banach space, we only need to verify that conditions (F1) and (F2) are satisfied.

Corollary 3.1 Assume that $E$ is an ordered and weakly sequentially complete Banach space, its positive cone $P$ is normal, and $-A$ generates a positive $C_{0}$-semigroup $\{T(t)\}_{t \geq 0}$ on $E, f \in C(J \times E \times E, E)$, and $u_{0} \in E$. If problem (1.1) has a lower solution $v_{0} \in C_{1-\gamma}(J, E)$ and an upper solution $w_{0} \in C_{1-\gamma}(J, E)$ with $v_{0} \leq w_{0}$. Suppose also that conditions (F0)(F4) are satisfied. Then problem (1.1) has minimal and maximal mild solutions $\underline{u}$ and $\bar{u}$ between $v_{0}$ and $w_{0}$, which can be obtained by a monotone iterative procedure starting from $v_{0}$ and $w_{0}$, respectively.

Proof In view of Theorem 3.1, if $E$ is weakly sequentially complete, then conditions (F3) and (F4) hold automatically. And by Theorem 2.2 in [23], any monotonic and order bounded sequence is precompact. By the monotonicity of (3.4), it is easy to see that $v_{n}(t)$ and $w_{n}(t)$ are convergent on $J$. Thus, $v_{n}(0)$ and $w_{n}(0)$ are convergent, i.e., condition (F4) holds. For $t \in J$, let $\left\{u_{n}\right\} \subset\left[v_{0}(t), w_{0}(t)\right]$ and $\left\{v_{n}\right\} \subset\left[G v_{0}, G w_{0}(t)\right]$ be two increasing or decreasing sequences. By (F2), $\left\{f\left(t, u_{n}, v_{n}\right)+C x_{n}\right\}$ is an ordered monotonic and ordered bounded sequence in $E$. Then $\alpha\left(\left\{f\left(t, u_{n}, v_{n}\right)+C x_{n}\right\}\right)=0$, (F3) holds, and by Theorem 3.1 our conclusion is valid.

Theorem 3.2 Assume that $E$ is an ordered Banach space, its positive cone $P$ is normal, and $-A$ generates a positive and equicontinuous $C_{0}$-semigroup $\{T(t)\}_{t \geq 0}$ on $E, f \in C(J \times$ $E \times E, E)$, and $u_{0} \in E$. If problem (1.1) has a lower solution $v_{0} \in C_{1-\gamma}(J, E)$ and an upper solution $w_{0} \in C_{1-\gamma}(J, E)$ with $v_{0} \leq w_{0}$. Suppose also that conditions (F0)-(F3) are satisfied and

(F5) There exists a nonnegative constant $L_{1}$ with

$$
\frac{2 M^{*} b^{\mu}\left(L_{1}+2 b L_{1} K_{0}+C\right)}{\Gamma(\mu)}\left[\frac{\left(b^{\gamma-1}-\Gamma(\gamma)\right) M^{*} \sum_{i=1}^{m} \lambda_{i}+\Gamma(\gamma)}{\Gamma(\gamma)\left(1-M^{*} \sum_{i=1}^{m} \lambda_{i}\right)}\right]<1
$$

such that

$$
\alpha\left(\left\{f\left(t, u_{n}, v_{n}\right)\right\}\right) \leq L_{1}\left(\alpha\left(\left\{u_{n}\right\}\right)+\alpha\left(\left\{v_{n}\right\}\right)\right)
$$

for $\forall t \in J$, and an equicontinuous countable set $\left\{u_{n}\right\} \subset\left[v_{0}(t), w_{0}(t)\right]$,

$\left\{v_{n}\right\} \subset\left[G v_{0}(t), G w_{0}(t)\right]$.

Then problem (1.1) has a minimal mild solution $\underline{u}$ and maximal mild solution $\bar{u}$ in $\left[v_{0}, w_{0}\right]$, and

$$
v_{n}(t) \rightarrow \underline{u}(t), \quad w_{n}(t) \rightarrow \bar{u}(t), \quad(n \rightarrow+\infty), t \in J,
$$

where $v_{n}(t)=Q v_{n-1}(t), w_{n}(t)=Q w_{n-1}(t)$, which satisfy

$$
v_{0}(t) \leq v_{1}(t) \leq \cdots v_{n}(t) \leq \cdots \underline{u}(t) \leq \bar{u}(t) \leq \cdots \leq w_{n}(t) \leq \cdots w_{1}(t) \leq w_{0}(t), \quad \forall t \in J .
$$


Proof From the proof of Theorem 3.1, we know that $Q:\left[v_{0}, w_{0}\right] \rightarrow\left[v_{0}, w_{0}\right]$ is continuous. First, we will prove that $Q:\left[v_{0}, w_{0}\right] \rightarrow C(J, E)$ is an equicontinuous operator. Since $T(t)(t \geq 0)$ is an equicontinuous $C_{0}$-semigroup, and $S(t)(t \geq 0)$ is also an equicontinuous $C_{0}$-semigroup, by the normality of the cone $P$, there exists $\bar{M}>0$ such that

$$
\|f(t, u(t), G u(t))+C u(t)\| \leq \bar{M}, \quad u \in\left[v_{0}, w_{0}\right]
$$

For any $u \in C_{1-\gamma}(J, E)$, let $y(t)=t^{1-\gamma} u(t)$, for $t_{1}=0,0<t_{2} \leq b$, we get

$$
\begin{aligned}
\| y\left(t_{2}\right) & -y(0) \| \\
\leq & \left\|t_{2}^{1-\gamma} S_{v, \mu}^{*}\left(t_{2}\right)\right\|\left(\Theta u_{0}\right)+\sum_{i=1}^{m} \lambda_{i} \Theta\left\|t_{2}^{1-\gamma} S_{v, \mu}^{*}\left(t_{2}\right)\right\| \int_{0}^{\tau_{i}} K_{\mu}^{*}\left(\tau_{i}-s\right) \\
& \times[f(s, u(s), G u(s))+C u(s)] d s \\
& +t_{2}^{1-\gamma}\left\|\int_{0}^{t_{2}} K_{\mu}^{*}\left(t_{2}-s\right)[f(s, u(s), G u(s))+C u(s)] d s\right\| \\
\leq & \left\|t_{2}^{1-\gamma} S_{v, \mu}^{*}\left(t_{2}\right)\right\|\left(\Theta u_{0}\right)+\bar{M} \sum_{i=1}^{m} \lambda_{i} \Theta\left\|t_{2}^{1-\gamma} S_{v, \mu}^{*}\left(t_{2}\right)\right\| \int_{0}^{\tau_{i}} K_{\mu}^{*}\left(\tau_{i}-s\right) d s \\
& +\bar{M}\left\|\int_{0}^{t_{2}} t_{2}^{1-\gamma} K_{\mu}^{*}\left(t_{2}-s\right) d s\right\| \\
\rightarrow & 0, \quad \text { as } t_{2} \rightarrow t_{1}=0 .
\end{aligned}
$$

For $0<t_{1}<t_{2} \leq b$, by (3.2), we get that

$$
\begin{aligned}
&\left\|y\left(t_{2}\right)-y\left(t_{1}\right)\right\| \\
& \leq\left\|t_{2}^{1-\gamma}(Q u)\left(t_{2}\right)-t_{1}^{1-\gamma}(Q u)\left(t_{1}\right)\right\| \\
& \leq\left\|t_{2}^{1-\gamma} S_{v, \mu}^{*}\left(t_{2}\right)-t_{1}^{1-\gamma} S_{v, \mu}^{*}\left(t_{1}\right)\right\|\left(\Theta u_{0}\right)+\left\|t_{2}^{1-\gamma} S_{v, \mu}^{*}\left(t_{2}\right)-t_{1}^{1-\gamma} S_{v, \mu}^{*}\left(t_{1}\right)\right\| \\
& \times \sum_{i=1}^{m} \lambda_{i} \Theta \int_{0}^{\tau_{i}} K_{\mu}^{*}\left(\tau_{i}-s\right)[f(s, u(s), G u(s))+C u(s)] d s \\
&+\int_{0}^{t_{2}} t_{2}^{1-\gamma} K_{\mu}^{*}\left(t_{2}-s\right)[f(s, u(s), G u(s))+C u(s)] d s \\
& \quad-\int_{0}^{t_{1}} t_{1}^{1-\gamma} K_{\mu}^{*}\left(t_{1}-s\right)[f(s, u(s), G u(s))+C u(s)] d s \\
& \leq\left(\left\|t_{2}^{1-\gamma} S_{v, \mu}^{*}\left(t_{2}\right)-t_{2}^{1-\gamma} S_{v, \mu}^{*}\left(t_{1}\right)\right\|\right. \\
&\left.+\left\|t_{2}^{1-\gamma} S_{v, \mu}^{*}\left(t_{1}\right)-t_{1}^{1-\gamma} S_{v, \mu}\left(t_{1}\right)\right\|\right)\left(\Theta u_{0}\right)+\left\|t_{2}^{1-\gamma} S_{v, \mu}^{*}\left(t_{2}\right)-t_{1}^{1-\gamma} S_{v, \mu}^{*}\left(t_{1}\right)\right\| \\
& \times \sum_{i=1}^{m} \lambda_{i} \Theta \int_{0}^{\tau_{i}} K_{\mu}^{*}\left(\tau_{i}-s\right)[f(s, u(s), G u(s))+C u(s)] d s \\
&+\left\|\int_{t_{1}}^{t_{2}} t_{2}^{1-\gamma} K_{\mu}^{*}\left(t_{2}-s\right)[f(s, u(s), G u(s))+C u(s)] d s\right\| \\
&+\| \int_{0}^{t_{1}} t_{2}^{1-\gamma} K_{\mu}^{*}\left(t_{2}-s\right)[f(s, u(s), G u(s))+C u(s)] d s
\end{aligned}
$$




$$
\begin{aligned}
& -\int_{0}^{t_{1}} t_{1}^{1-\gamma} K_{\mu}^{*}\left(t_{2}-s\right)[f(s, u(s), G u(s))+C u(s)] d s \| \\
& +\| \int_{0}^{t_{1}} t_{1}^{1-\gamma} K_{\mu}^{*}\left(t_{2}-s\right)[f(s, u(s), G u(s))+C u(s)] d s \\
& -\int_{0}^{t_{1}} t_{1}^{1-\gamma} K_{\mu}^{*}\left(t_{1}-s\right)[f(s, u(s), G u(s))+C u(s)] d s \| \\
& =J_{1}+J_{2}+J_{3}+J_{4}+J_{5}+J_{6},
\end{aligned}
$$

where

$$
\begin{aligned}
J_{1}= & \left(\left\|t_{2}^{1-\gamma} S_{v, \mu}^{*}\left(t_{2}\right)-t_{2}^{1-\gamma} S_{v, \mu}^{*}\left(t_{1}\right)\right\|\right)\left(\Theta u_{0}\right) \\
J_{2}= & \left(\left\|t_{2}^{1-\gamma} S_{v, \mu}^{*}\left(t_{1}\right)-t_{1}^{1-\gamma} S_{v, \mu}^{*}\left(t_{1}\right)\right\|\right)\left(\Theta u_{0}\right) \\
J_{3}= & \left\|t_{2}^{1-\gamma} S_{v, \mu}^{*}\left(t_{2}\right)-t_{1}^{1-\gamma} S_{v, \mu}^{*}\left(t_{1}\right)\right\| \sum_{i=1}^{m} \lambda_{i} \Theta \int_{0}^{\tau_{i}} K_{\mu}^{*}\left(\tau_{i}-s\right)[f(s, u(s), G u(s))+C u(s)] d s, \\
J_{4}= & \left\|\int_{t_{1}}^{t_{2}} t_{2}^{1-\gamma} K_{\mu}^{*}\left(t_{2}-s\right)[f(s, u(s), G u(s))+C u(s)] d s\right\| \\
J_{5}= & \| \int_{0}^{t_{1}} t_{2}^{1-\gamma} K_{\mu}^{*}\left(t_{2}-s\right)[f(s, u(s), G u(s))+C u(s)] d s \\
& -\int_{0}^{t_{1}} t_{1}^{1-\gamma} K_{\mu}^{*}\left(t_{2}-s\right)[f(s, u(s), G u(s))+C u(s)] d s \| \\
J_{6}= & \| \int_{0}^{t_{1}} t_{1}^{1-\gamma} K_{\mu}^{*}\left(t_{2}-s\right)[f(s, u(s), G u(s))+C u(s)] d s \\
& -\int_{0}^{t_{1}} t_{1}^{1-\gamma} K_{\mu}^{*}\left(t_{1}-s\right)[f(s, u(s), G u(s))+C u(s)] d s \| .
\end{aligned}
$$

Here we calculate

$$
\left\|t_{2}^{1-\gamma}(Q u)\left(t_{2}\right)-t_{1}^{1-\gamma}(Q u)\left(t_{1}\right)\right\| \leq \sum_{i=1}^{6}\left\|J_{i}\right\| .
$$

Therefore, it is not difficult to see that $\left\|J_{i}\right\|$ tends to 0 , when $t_{2}-t_{1} \rightarrow 0, i=1,2, \ldots, 6$.

For $J_{1}$, by Lemma 2.10, we get

$$
\begin{aligned}
J_{1} & =\left(\left\|t_{2}^{1-\gamma} S_{v, \mu}^{*}\left(t_{2}\right)-t_{2}^{1-\gamma} S_{v, \mu}^{*}\left(t_{1}\right)\right\|\right)\left(\Theta u_{0}\right) \\
& \leq\left\|t_{2}^{1-\gamma}\left(S_{v, \mu}^{*}\left(t_{2}\right)-S_{v, \mu}\left(t_{1}\right)\right)\right\|\left(\Theta u_{0}\right) \rightarrow 0, \quad \text { as } t_{2} \rightarrow t_{1} .
\end{aligned}
$$

For $J_{2}$, by Lemma 2.10, we get

$$
\begin{aligned}
J_{2} & =\left(\left\|t_{2}^{1-\gamma} S_{v, \mu}^{*}\left(t_{1}\right)-t_{1}^{1-\gamma} S_{v, \mu}^{*}\left(t_{1}\right)\right\|\right)\left(\Theta u_{0}\right) \\
& \leq \frac{M^{*} b^{\gamma-1}}{\Gamma(\gamma)}\left\|t_{2}^{1-\gamma}-t_{1}^{1-\gamma}\right\|\left\|\Theta u_{0}\right\| \\
& \leq \frac{M^{*} b^{\gamma-1}}{\Gamma(\gamma)}\left\|\left(t_{2}-t_{1}\right)^{1-\gamma}\right\|\left\|\Theta u_{0}\right\| \rightarrow 0, \quad \text { as } t_{2} \rightarrow t_{1} .
\end{aligned}
$$


For $J_{3}$, by Lemma 2.10 , we have

$$
\begin{aligned}
J_{3} & =\sum_{i=1}^{m} \lambda_{i} \Theta\left\|t_{2}^{1-\gamma} S_{v, \mu}^{*}\left(t_{1}\right)-t_{1}^{1-\gamma} S_{v, \mu}^{*}\left(t_{1}\right)\right\| \int_{0}^{\tau_{i}} K_{\mu}^{*}\left(\tau_{i}-s\right)[f(s, u(s), G u(s))+C u(s)] d s \\
& \leq \frac{\bar{M} \sum_{i=1}^{m}\left|\lambda_{i}\right|}{1-M^{*} \sum_{i=1}^{m}\left|\lambda_{i}\right|}\left\|t_{2}^{1-\gamma} S_{v, \mu}^{*}\left(t_{1}\right)-t_{1}^{1-\gamma} S_{v, \mu}^{*}\left(t_{1}\right)\right\| \int_{0}^{\tau_{i}} K_{\mu}^{*}\left(\tau_{i}-s\right) d s \\
& \rightarrow 0, \quad \text { as } t_{2} \rightarrow t_{1} .
\end{aligned}
$$

For $J_{4}$, by Lemma 2.10 , we have

$$
\begin{aligned}
J_{4} & =\left\|\int_{t_{1}}^{t_{2}} t_{2}^{1-\gamma} K_{\mu}^{*}\left(t_{2}-s\right)[f(s, u(s), G u(s))+C u(s)] d s\right\| \\
& \leq \bar{M} \int_{t_{1}}^{t_{2}} t_{2}^{1-\gamma} K_{\mu}^{*}\left(t_{2}-s\right) d s \\
& \rightarrow 0, \quad \text { as } t_{2} \rightarrow t_{1} .
\end{aligned}
$$

For $J_{5}$, by Lemma 2.10 , we have

$$
\begin{aligned}
J_{5}= & \| \int_{0}^{t_{1}} t_{2}^{1-\gamma} K_{\mu}^{*}\left(t_{2}-s\right)[f(s, u(s), G u(s))+C u(s)] d s \\
& -\int_{0}^{t_{1}} t_{1}^{1-\gamma} K_{\mu}^{*}\left(t_{2}-s\right)[f(s, u(s), G u(s))+C u(s)] d s \| \\
\leq & \frac{2 M^{*}}{\Gamma(\mu)} \int_{0}^{t_{1}}\left[t_{2}^{1-\gamma}\left(t_{2}-s\right)^{\mu-1}-t_{1}^{1-\gamma}\left(t_{1}-s\right)^{\mu-1}\right][f(s, u(s), G u(s))+C u(s)] d s
\end{aligned}
$$

Noting that

$$
\begin{aligned}
& \int_{0}^{t_{1}}\left[t_{2}^{1-\gamma}\left(t_{2}-s\right)^{\mu-1}-t_{1}^{1-\gamma}\left(t_{1}-s\right)^{\mu-1}\right][f(s, u(s), G u(s))+C u(s)] d s \\
& \quad \leq \int_{0}^{t_{1}} t_{2}^{1-\gamma}\left(t_{2}-s\right)^{\mu-1}[f(s, u(s), G u(s))+C u(s)] d s
\end{aligned}
$$

and

$$
\int_{0}^{t_{1}}\left[t_{2}^{1-\gamma}\left(t_{2}-s\right)^{\mu-1}-t_{1}^{1-\gamma}\left(t_{1}-s\right)^{\mu-1}\right][f(s, u(s), G u(s))+C u(s)] d s
$$

exists, and by the Lebesgue dominated convergence theorem, we have

$$
\begin{aligned}
& \int_{0}^{t_{1}}\left[t_{2}^{1-\gamma}\left(t_{2}-s\right)^{\mu-1}-t_{1}^{1-\gamma}\left(t_{1}-s\right)^{\mu-1}\right][f(s, u(s), G u(s))+C u(s)] d s \\
& \quad \rightarrow 0, \quad \text { as } t_{2} \rightarrow t_{1} .
\end{aligned}
$$

It is easy to see that $\lim _{t_{2} \rightarrow t_{1}} J_{5}=0$. 
For $J_{6}$, by Lemma 2.10, we have

$$
\begin{aligned}
J_{6}= & \| \int_{0}^{t_{1}} t_{1}^{1-\gamma} K_{\mu}^{*}\left(t_{2}-s\right)[f(s, u(s), G u(s))+C u(s)] d s \\
& -\int_{0}^{t_{1}} t_{1}^{1-\gamma} K_{\mu}^{*}\left(t_{1}-s\right) f(s, u(s)) d s \| \\
\leq & \left\|K_{\mu}^{*}\left(t_{2}-s\right)-K_{\mu}^{*}\left(t_{1}-s\right)\right\| \int_{0}^{t_{1}} t_{1}^{1-\gamma}[f(s, u(s), G u(s))+C u(s)] d s \\
\rightarrow & 0, \quad \text { as } t_{2} \rightarrow t_{1} .
\end{aligned}
$$

In conclusion,

$$
\left\|y\left(t_{2}\right)-y\left(t_{1}\right)\right\| \leq\left\|t_{2}^{1-\gamma}(Q u)\left(t_{2}\right)-t_{1}^{1-\gamma}(Q u)\left(t_{1}\right)\right\| \rightarrow 0
$$

as $t_{2} \rightarrow t_{1}$, i.e.,

$$
\left\|(Q u)\left(t_{2}\right)-(Q u)\left(t_{1}\right)\right\|_{\gamma} \rightarrow 0, \quad \text { as } t_{2} \rightarrow t_{1},
$$

which means that $Q:\left[v_{0}, w_{0}\right] \rightarrow\left[v_{0}, w_{0}\right]$ is equicontinuous.

So, for any $D \subset\left[v_{0}, w_{0}\right], Q(D) \subset\left[v_{0}, w_{0}\right]$ is bounded and equicontinuous. Therefore, by Lemma 2.2, there exists a countable set $D_{0}=\left\{u_{n}\right\} \subset D$ such that

$$
\alpha(Q(D)) \leq 2 \alpha\left(Q\left(D_{0}\right)\right)
$$

For $t \in J$, by the definition of the operator $Q$, we have

$$
\begin{aligned}
\alpha\left(Q\left(D_{0}(t)\right)\right) & \alpha\left(\left\{S_{v, \mu}^{*}(t) \Theta u_{0}+\sum_{i=1}^{m} \lambda_{i} S_{v, \mu}^{*}(t) \Theta \int_{0}^{\tau_{i}} K_{\mu}^{*}\left(\tau_{i}-s\right)\left[f\left(s, v_{n-1}(s), G v_{n-1}(s)\right)\right.\right.\right. \\
= & \left.\left.\left.+C v_{n-1}(s)\right] d s+\int_{0}^{t} K_{\mu}^{*}(t-s)\left[f\left(s, v_{n-1}(s), G v_{n-1}(s)\right)+C v_{n-1}(s)\right] d s\right\}\right) \\
\leq & \frac{2\left(M^{*}\right)^{2} \sum_{i=1}^{m} \lambda_{i} b^{\mu+\gamma-2}\left(L_{1}+2 b L_{1} K_{0}+C\right)}{\Gamma(\gamma) \Gamma(\mu)\left(1-M^{*} \sum_{i=1}^{m}\right)} \int_{0}^{\tau_{i}} \alpha\left(D_{0}(s)\right) d s \\
& +\frac{2 M^{*} b^{\mu-1}\left(L_{1}+2 b L_{1} K_{0}+C\right)}{\Gamma(\mu)} \int_{0}^{t} \alpha\left(D_{0}(s)\right) d s \\
\leq & \frac{2\left(M^{*}\right)^{2} \sum_{i=1}^{m} \lambda_{i} b^{\mu+\gamma-1}\left(L_{1}+2 b L_{1} K_{0}+C\right)}{\Gamma(\gamma) \Gamma(\mu)\left(1-\sum_{i=1}^{m} \lambda_{i}\right)} \alpha(D)+\frac{2 M^{*} b^{\mu}\left(L_{1}+2 b L_{1} K_{0}+C\right)}{\Gamma(\mu)} \alpha(D) \\
\leq & \frac{2 M^{*} b^{\mu}\left(L_{1}+2 b L_{1} K_{0}+C\right)}{\Gamma(\mu)}\left[\frac{b^{\gamma-1} M^{*} \sum_{i=1}^{m} \lambda_{i}}{\Gamma(\gamma)\left(1-\sum_{i=1}^{m} \lambda_{i}\right)}+1\right] \alpha(D) \\
= & \frac{2 M^{*} b^{\mu}\left(L_{1}+2 b L_{1} K_{0}+C\right)}{\Gamma(\mu)}\left[\frac{\left.\left(b^{\gamma-1}-\Gamma(\gamma)\right) M^{*} \sum_{i=1}^{m} \lambda_{i}+\Gamma(\gamma)\right] \alpha(D)}{\Gamma(\gamma)\left(1-M^{*} \sum_{i=1}^{m} \lambda_{i}\right)}\right]
\end{aligned}
$$

Since $Q\left(D_{0}\right)$ is bounded and equicontinuous, we know from Lemma 2.3 that

$$
\alpha\left(Q\left(D_{0}\right)\right)=\max _{t \in I} \alpha\left(Q\left(D_{0}\right)(t)\right)
$$


And by (3.6), we have

$$
\alpha(Q(D)) \leq \eta \alpha(D)
$$

where

$$
\eta=\frac{2 M^{*} b^{\mu}\left(L_{1}+2 b L_{1} K_{0}+C\right)}{\Gamma(\mu)}\left[\frac{\left(b^{\gamma-1}-\Gamma(\gamma)\right) M^{*} \sum_{i=1}^{m} \lambda_{i}+\Gamma(\gamma)}{\Gamma(\gamma)\left(1-M^{*} \sum_{i=1}^{m} \lambda_{i}\right)}\right]<1 .
$$

Thus, $Q:\left[v_{0}, w_{0}\right] \rightarrow\left[v_{0}, w_{0}\right]$ is a condensing operator. By Lemma 2.11, our conclusion is valid.

\section{Applications}

In this section, we present an example that illustrates the applicability of our main results.

Example 4.1 We consider the following fractional partial differential equation:

$$
\left\{\begin{array}{l}
D_{0+}^{v, \mu} u(t, x)=\sum_{|\alpha| \leq 2 m} a_{\alpha} D_{x}^{\alpha} u(t, x)+f(t, x, u(t, x), G u(t, x)), \quad(t, x) \in J \times \Omega \\
I_{0+}^{(1-v)(1-\mu)} u(0, x)=u_{0}+\sum_{i=1}^{m} \lambda_{i} u\left(\tau_{i}, x\right)
\end{array}\right.
$$

where $D_{0+}^{v, \mu}$ is the Hilfer fractional derivative, $0 \leq v \leq 1,0<\mu<1, t \in J=[0, b], \lambda_{i} \neq 0$, $i=1,2, \ldots, m$, integer $\mathbb{N} \geq 1, \Omega \subset \mathbb{R}^{N}$ is a bounded domain with a sufficiently smooth boundary $\partial \Omega, f: J \times E \times E \rightarrow E$ is continuous and

$$
D_{x}^{\alpha}=\left(\frac{\partial}{\partial x_{1}}\right)^{\alpha_{1}}\left(\frac{\partial}{\partial x_{2}}\right)^{\alpha_{2}} \cdots\left(\frac{\partial}{\partial x_{n}}\right)^{\alpha_{n}}
$$

$\alpha=\left(\alpha_{1}, \alpha_{2}, \ldots, \alpha_{n}\right)$ is an $n$-dimensional multi-index, $|\alpha|=\alpha_{1}+\alpha_{2}+\cdots+\alpha_{n}$, coefficient function $a_{\alpha}(x) \in C^{2 m}(\bar{\Omega})$.

Let $E=L^{p}(\Omega)$ with $1<p<\infty, P=\left\{u \in L^{p}(\Omega)\right\}: u(x) \geq 0$, q.e. $\left.x \in \Omega\right\}$, and define the operator $A: D(A) \subset E \rightarrow E$ as follows:

$$
D(A)=W^{2 m, p} \cap W_{0}^{m, p}(\Omega), \quad A u=\sum_{|\alpha| \leq 2 m} a_{\alpha} D_{x}^{\alpha} u
$$

Then $E$ is a Banach space, $P$ is a normal cone of $E$, and $-A$ generates a positive $C_{0}$ semigroup $T(t)(t \geq 0)$ in $E$ (see [21]). Let $f(t, u(t), G u(t))=f(t, x, u(t, x), G u(t, x)), u_{0}=$ $u_{0}(\cdot)$, then problem (4.1) can be written as abstract (1.1).

Theorem 4.1 If the following conditions are satisfied:

(H1) Let $u_{0}(x) \geq 0, x \in \Omega$, and there exists a function $w=w(t, x) \in C_{1-\gamma}(J \times \Omega)$ such that

$$
\left\{\begin{array}{l}
D_{0+}^{v, \mu} u(t, x) \geq \sum_{|\alpha| \leq 2 m} a_{\alpha} D_{x}^{\alpha} u(t, x)+f(t, x, u(t, x), G u(t, x)) \\
I_{0+}^{(1-v)(1-\mu)} u(0, x)=u_{0}+\sum_{i=1}^{m} \lambda_{i} u\left(\tau_{i}, x\right)
\end{array}\right.
$$


(H2) There exists a constant $M>0$ such that

$$
f\left(t, x, u_{2}, v_{2}\right)-f\left(t, x, u_{1}, v_{1}\right) \geq-M\left(u_{2}-u_{1}\right)
$$

for any $t \in J$, and $0 \leq u_{1} \leq u_{2} \leq w(t, x), 0 \leq v_{1} \leq v_{2} \leq G w(t, x)$.

(H3) $\lambda_{i}>0(i=1,2, \ldots, m)$ and $\sum_{i=1}^{m} \lambda_{i}<\frac{\Gamma(\gamma)}{M^{*} b^{\gamma-1}}$.

(H4) There exists a constant $L>0$ such that

$$
\alpha\left(\left\{f\left(t, u_{n}, v_{n}\right)\right\}\right) \leq L\left(\alpha\left(\left\{u_{n}\right\}\right)+\alpha\left(\left\{v_{n}\right\}\right)\right)
$$

for $\forall t \in J$, and increasing or decreasing monotonic sequences $\left\{u_{n}\right\} \subset\left[v_{0}(t), w_{0}(t)\right]$ and $\left\{v_{n}\right\} \subset\left[G v_{0}(t), G w_{0}(t)\right]$.

Then problem (4.1) has minimal and maximal mild solutions between 0 and $w(x, t)$, which can be obtained by a monotone iterative procedure starting from 0 and $w(t)$, respectively.

Proof Assumption (H1) implies that $v_{0} \equiv 0$ and $w_{0} \equiv w(x, t)$ are lower and upper solutions of problem (4.1), respectively, and from (H2), it is easy to verify that all conditions (F1)-(F3) are satisfied under the constant $M=1$. So our conclusion follows from Theorem 3.1.

\section{Conclusions}

The purpose of this paper was to obtain existence results of mild solutions for a class of evolution equations with Hilfer fractional derivative. The method is inspired by using the fixed point theorem combined with the method of lower and upper solutions, some existence result of mild solutions for Hilfer fractional evolution equations with nonlocal conditions has been obtained. Here, we do not require that $C_{0}$-semigroup $\{T(t)\}_{t \geq 0}$ is compact.

\section{Acknowledgements}

The authors would like to thank the referees for their useful suggestions that have significantly improved the paper. Supported by the National Natural Science Foundation of China (Grant No. 11661071).

\section{Funding}

The authors are supported financially by the National Natural Science Foundation of China (11661071)

Availability of data and materials

Data sharing not applicable to this article as no datasets were generated or analysed during the current study.

Ethics approval and consent to participate

Not applicable.

Competing interests

The authors declare that there is no conflict of interests regarding the publication of this paper.

Authors' contributions

All authors contributed equally and significantly in writing this article. All authors read and approved the final manuscript.

\section{Publisher's Note}

Springer Nature remains neutral with regard to jurisdictional claims in published maps and institutional affiliations. 


\section{References}

1. Hilfer, R.: Applications of Fractional Calculus in Physics. World Scientific, Singapore (2000)

2. Gu, H., Trujillo, J.J.: Existence of mild solution for evolution equation with Hilfer fractional derivative. Appl. Math. Comput. 257, 344-354 (2015)

3. Yang, M., Wang, Q.: Existence of mild solutions for a class of Hilfer fractional evolution equations with nonlocal conditions. Fract. Calc. Appl. Anal. 20(3), 679-705 (2017)

4. Hilfer, R.: In: Hilfer, R. (ed.) Fractional Time Evolution, Applications of Fractional Calculus in Physics, pp. 87-130. World Scientific, Singapore (2000)

5. Ahmed, H.M., El-Borai, M.M.: Hilfer fractional stochastic integro-differential equations. Appl. Math. Comput. 331, $182-189(2018)$

6. Ahmed, H.M., El-Borai, M.M., El-Owaidy, H.M., Ghanem, A.S.: Impulsive Hilfer fractional differential equations. Adv. Differ. Equ. 2018, 226 (2018)

7. Gou, H., Li, B.: Study on the mild solution of Sobolev type Hilfer fractional evolution equations with boundary conditions. Chaos Solitons Fractals 112, 168-179 (2018)

8. Furati, K.M., Kassim, M.D., Tatar, N.e-.: Existence and uniqueness for a problem involving Hilfer factional derivative. Comput. Math. Appl. 64, 1616-1626 (2012)

9. $\mathrm{Mu}$, J., Li, Y.: Monotone interactive technique for impulsive fractional evolution equations. J. Inequal. Appl. 2011,125 (2011)

10. $\mathrm{Mu}, \mathrm{J}$.: Extremal mild solutions for impulsive fractional evolution equations with nonlocal initial conditions. Bound. Value Probl. 2012, 71 (2012)

11. Liang, J., Yang, H.: Controllability of fractional integro-differential evolution equations with nonlocal conditions. Appl. Math. Comput. 254, 20-29 (2015)

12. $\mathrm{Mu}, \mathrm{J}$.: Monotone iterative technique for fractional evolution equations in Banach spaces. J. Appl. Math. 2011, Article ID $767186(2011)$

13. Shu, X.B., Xu, F.: Upper and lower solution method for fractional evolution equations with order $1<\alpha<2$. J. Korean Math. Soc. 51(6), 1123-1139(2014)

14. Chen, P., Li, Y.: Monotone iterative technique for a class of semilinear evolution equations with nonlocal conditions. Results Math. 63, 731-744 (2013)

15. Singh, V., Pandey, D.N.: A study of Sobolev type fractional impulsive differential systems with fractional nonlocal conditions. Int. J. Appl. Comput. Math. 4, 12 (2018)

16. Li, F., Liang, J., Xu, H.: Existence of mild solutions for fractional integrodifferential equations of Sobolev type with nonlocal conditions. J. Math. Anal. Appl. 391, 510-525 (2012)

17. Liu, L.S., Guo, F., Wu, C.X., Wu, Y.H.: Existence theorems of global solutions for nonlinear Volterra type integral equations in Banach spaces. J. Math. Anal. Appl. 309, 638-649 (2005)

18. Li, Y.: The positive solutions of abstract semilinear evolution equations and their applications. Acta Math. Sin. 39(5), 666-672 (1996) (in Chinese)

19. Guo, D., Sun, J.: In: Ordinary Differential Equations in Abstract Spaces. Shandong Science and Technology, Jinan (1989) (in Chinese)

20. Heinz, H.R.: On the behavior of measure of noncompactness with respect to differentiation and integration of vector-valued functions. Nonlinear Anal. 71, 1351-1371 (1983)

21. Pazy, A.: Semigroups of Linear Operators and Applications to Partial Differential Equations. Springer, Berlin (1983)

22. Ye, H., Gao, J., Ding, Y.: A generalized Gronwall inequality and its applications to a fractional differential equation. J. Math. Anal. Appl. 328, 1075-1081 (2007)

23. Du, Y.: Fixed points of increasing operators in order Banach spaces and applications. Appl. Anal. 38, 1-20 (1990)

\section{Submit your manuscript to a SpringerOpen ${ }^{\circ}$ journal and benefit from:}

- Convenient online submission

- Rigorous peer review

- Open access: articles freely available online

- High visibility within the field

- Retaining the copyright to your article

Submit your next manuscript at $\boldsymbol{s p r i n g e r o p e n . c o m ~}$ 